\title{
Circularly Polarized Microstrip Antenna with Frequency Of 1.9- 2.5 Ghz For Wireless Applications
}

\author{
Kamal Mohamed Elsanoussi ${ }^{1}$, Onny Setyawati ${ }^{2}$, Muladi Muladi ${ }^{3}$ \\ ${ }^{1}$ Student of Master Program, Department of Electrical Engineering, Faculty of Engineering, Brawijaya \\ University, Malang, Indonesia \\ ${ }^{2}$ Department of Electrical Engineering, Faculty of Engineering, Brawijaya University, Malang, Indonesia \\ ${ }^{3}$ Department of Electrical Engineering, Faculty of Engineering, State university of Malang, Indonesia
}

\begin{abstract}
Wireless application can work better if the supporting component especially the antenna can transmit and receive the power with low losses due to the misalignment. Reducing the power losses caused by misalignment can be performed with the circularly polarized antenna. We proposed the circularly polarized antenna, with $40 \mathrm{~mm} \times 40 \mathrm{~mm}$ in dimension and FR-4 substrate with the dielectric constant of 4.4. The microstrip line fed and the power divider method were used to get orthogonal mode. From the measurement result, the antenna can work at frequency range of 1.9 up to $2.5 \mathrm{GHz}$ omnidirectionally and has an axial ratio value below $3 \mathrm{~dB}$.
\end{abstract}

Keyword: Circularly Polarized; Microstrip Antenna

\section{Introduction}

Many antennas can be selected for wireless applications. One of them is microstrip antennas. The advantages of microstip antennas are technically simple to be fabricated and they are lightweight and small-size [2]. If the microstrip antenna is used for wireless technology, it can be affected on dimension of wireless device. However, the microstrip antennas also have a disadvantages such as narrow bandwith [2]. The microstrip antenna still can be developed by increasing its bandwidth until $70 \%$ to obtain the broadband antenna [2].

Broadband microstrip antenna works using wide band frequency. The antenna has many advantages, such as low power consumption, low cost construction and can transmit information with high data rate [4]; hence broadband microstrip antenna work as a multi-purpose antenna in communication system.

Nowadays, the sizes of the broadband microstrip antennas become smaller due to miniaturization of wireless system components. Although the size of antenna is reduced, the performance of the antenna, especially at operation frequency still can be increased. And also the liability to receive and transmit maximum radiated power which is influenced by the polarization of the antenna.

There are 3 types of the antenna polarization, i.e. linear polarization, elliptical polarization and circular polarization. From all of them, the Circularly Polarized (CP) is required for antenna to reduce lossesdue to mismatched between signal and the receiving antenna [5] and $\mathrm{CP}$ can rise the flexibility of antenna pointing. It is particulary useful for Radar, communication and navigation systems, because of the direction of the alignment between transmitter and receiver antenna can be abandoned [2]. Also, CP antenna is still being developed to get better antenna performance than existing antenna. However, the circularly polarized antennas have complexity on design because circularly polarized antennas have several terms, such as the antenna must have two modes which have phase difference of $90^{\circ}$ by modifying the antenna patch design shape with one or more feeds [1].

Several related journal to this circularly polarized microstrip antenna are published, such as reported by Eman and Darwish [5] about circularly polarized microstrip antenna with the tuning fork for ultra-wideband. The antenna worked at 3.8-17.6 GHz with the dimension of $32 \times 28.1 \mathrm{~mm}$ and the thickness of $0.8 \mathrm{~mm}$ and made of FR-4 Epoxy material. Reported from Chen, Zheng, Quan and Li [6] on their research about the circularly polarized antenna with equiangular tapered-shaped feed line for ultra-wideband application which was made with coplanar waveguide fed printed on FR-4 epoxy by the size of $60 \mathrm{~mm} \times 60 \mathrm{~mm}$ and worked at frequency of 2.9-20 GHz, and circularly polarized frequency at 2.9-5.5 GHz. Also, Joseph and Fukusako [6] presented about circularly polarized broadband antenna with a circular slot on a circular ground plane. The antenna was printed on Arlon diclad 522 substrate within diameter of $80 \mathrm{~mm}$, and thickness of $0.8 \mathrm{~mm}$. The antenna worked at the frequency of 3-4.9 GHz and at circularly polarized frequency of 2.81-4.6 GHz.

By designing and constructing the circularly polarized microstrip antenna, the problem on propagation can be minimized. Not only that, if the antenna can be worked at $1.9 \mathrm{GHz}$ until $2.5 \mathrm{GHz}$, the antenna will be able to provide several wireless applications such as PCS $(1.9 \mathrm{GHz})$ and Wifi $(2.4 \mathrm{GHz})$. 


\section{Antenna Design And Fabrication}

The microstrip antenna was designed on FR-4 Epoxy material which has dielectric constant $\left(\varepsilon_{\mathrm{r}}\right)$ of 4.4 with the thickness of the substrate $0.8 \mathrm{~mm}$. The size of the radiator of the microstrip antenna can be determined by making its size proportional to the substrate size. Meanwhile, the width of the microstrip-line can be obtained from the Equation 1 [7].

$$
\frac{w}{h}=\frac{8 \exp (A)}{\exp (2 A)-2}
$$

Which A is defined as:

$$
A=\frac{Z_{0}}{60}\left(\frac{\varepsilon_{r}+1}{2}\right)^{1 / 2}+\frac{\varepsilon_{r}-1}{\varepsilon_{r}+1}\left(0.23+\frac{0.11}{\varepsilon_{r}}\right)
$$

Where $\mathrm{w}$ is the width of the microstrip line in meter, $\mathrm{h}$ is the thickness of the substrate in meter, $\mathrm{Z}_{0}$ is the characteristic impedance in ohm, and $\varepsilon_{\mathrm{r}}$ is the dielectric constant of the substrate.

The characteristic impedance $\left(\mathrm{Z}_{0}\right)$ is defined as $50 \mathrm{ohm}$. The value of characteristic impedance is taken from technical specification of SMA connector of the antenna, hence A can be defined as

$$
\begin{aligned}
& A=\frac{50}{60}\left(\frac{4.4+1}{2}\right)^{1 / 2}+\frac{4.4-1}{4.4+1}\left(0.23+\frac{0.11}{4.4}\right) \\
& A=1.5
\end{aligned}
$$
follows

After A is defined, the ratio of the microstrip line width and the substrate's thickness can be defined as

$$
\begin{aligned}
\frac{w}{h} & =\frac{8 \exp (1.5)}{\exp (2(1.5))-2} \\
\frac{w}{h} & =1.9
\end{aligned}
$$
follow

The thickness of the substrate is defined $0.8 \mathrm{~mm}$, hence the width of the microstrip line is obtainec

$$
\begin{aligned}
& \frac{w}{h}=1.9 \\
& \frac{w}{0.0008}=1.9 \\
& w=0.0015 \quad \text { meter }
\end{aligned}
$$

Hence, the antenna design is shown in Figure 1

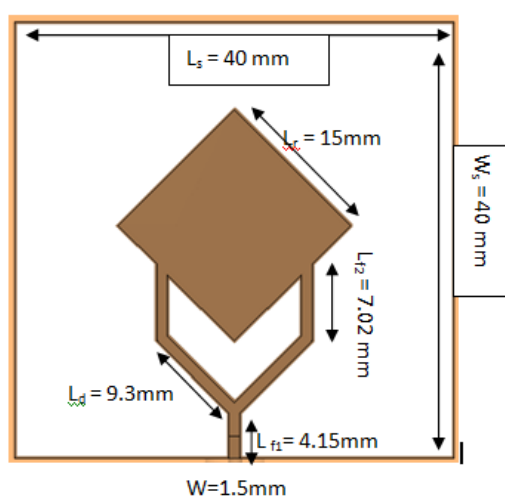

(a)

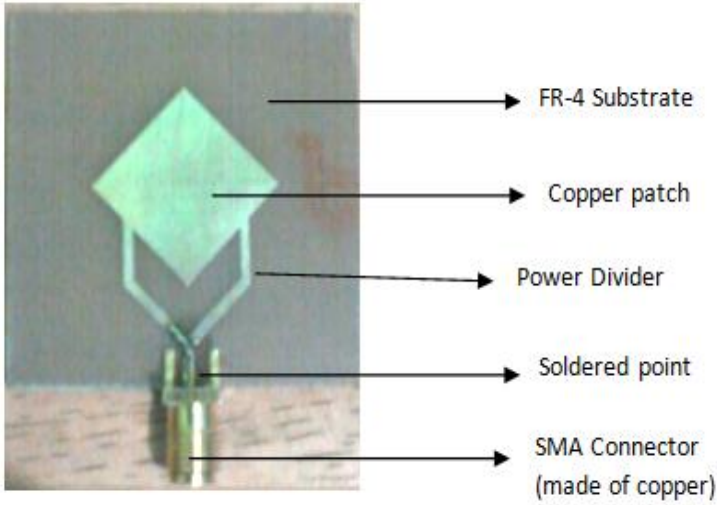

(b)

Fig 1 (a) The antenna design; (b) The fabricated antenna 
The dimension of the antenna especially the size of the substrate, the width of the radiator, and the length of the divider can be changed and optimized. Hence, the substrate can be defined as $40 \mathrm{~mm} \times 40 \mathrm{~mm}$. The radiator was $15 \mathrm{~mm} \times 15 \mathrm{~mm}$. The feed line which connected the divider and the radiator had $7.02 \mathrm{~mm}$ in length, wherein each of the divider line had $9.3 \mathrm{~mm}$ in length and the main feedline had $4.15 \mathrm{~mm}$ in length.

Fabricating the antenna used defined material which is FR-4 Epoxy with dielectric constant of 4.4 with the dimension of the antenna as obtained in Figure 1. The antenna was fabricated with the microstrip-line fed and used power divider to get circular polarization. The fabricated antenna is shown in Figure 1.

\subsection{S-Parameter $\left(S_{11}\right)$ Measurement Result}

\section{Measurement Result}

The measurement of the S-Parameter or $S_{11}$ was the basic parameter to obtain the work frequency of the antenna, by determining the reflected power level of the antenna due to mismatched impedance condition on the transmission line. $\mathrm{S}_{11}$ parameter's measurement needed a spectrum analyzer and a directional coupler to get the result with the step input connector of the directional coupler was connected to output connector of the spectrum analyzer and the antenna was connected to output connector of the directional coupler. Meanwhile, the coupler connector was connected to input connector of the spectrum analyzer. The measurement setup and results are shown in Figure 3.

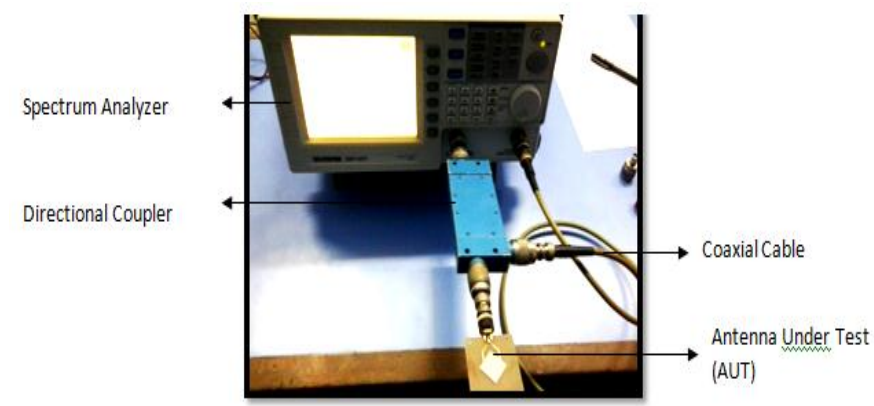

Fig. $2 \mathrm{~S}_{11}$ measurement setup

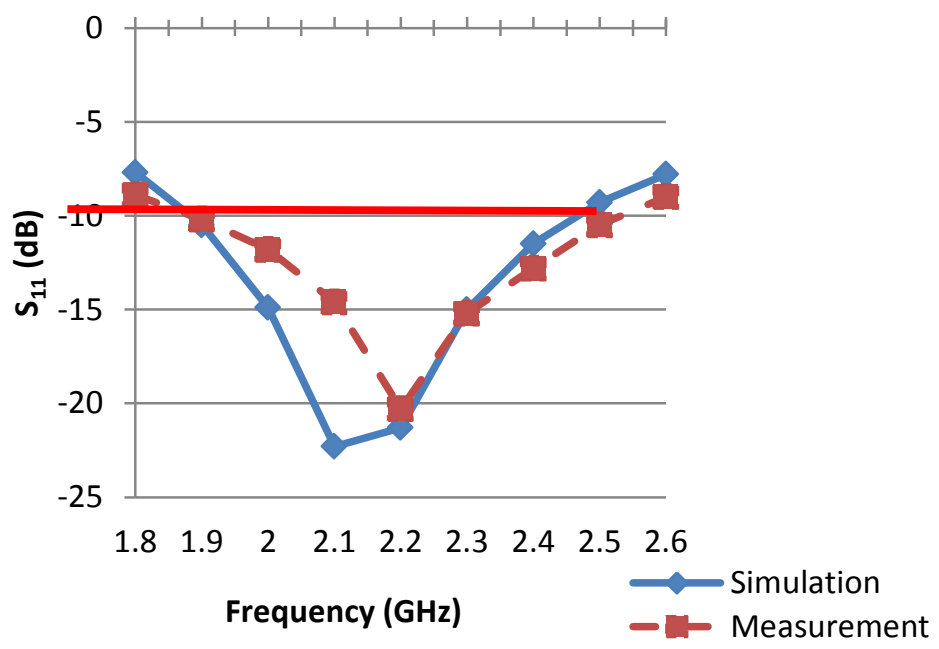

Fig. $3 \mathrm{~S}_{11}$ measurement setup and result

Figure 3 shows that the antenna could work at the frequency range of 1.9 until $2.5 \mathrm{GHz}$. The measured $\mathrm{S}_{11}$ had level below $-9.54 \mathrm{~dB}$ at frequency of 1.9 until $2.5 \mathrm{GHz}$. The result between simulation and measurement were similar in the working frequency range even though there were several different values of $S_{11}$ level. The differences occurred due to the losses on the connector in the measurement device, and the calibration of the device.

\subsection{Gain Measurement Result}

The graphic of the antenna gain from measurement and simulation results are shown in Figure 4 


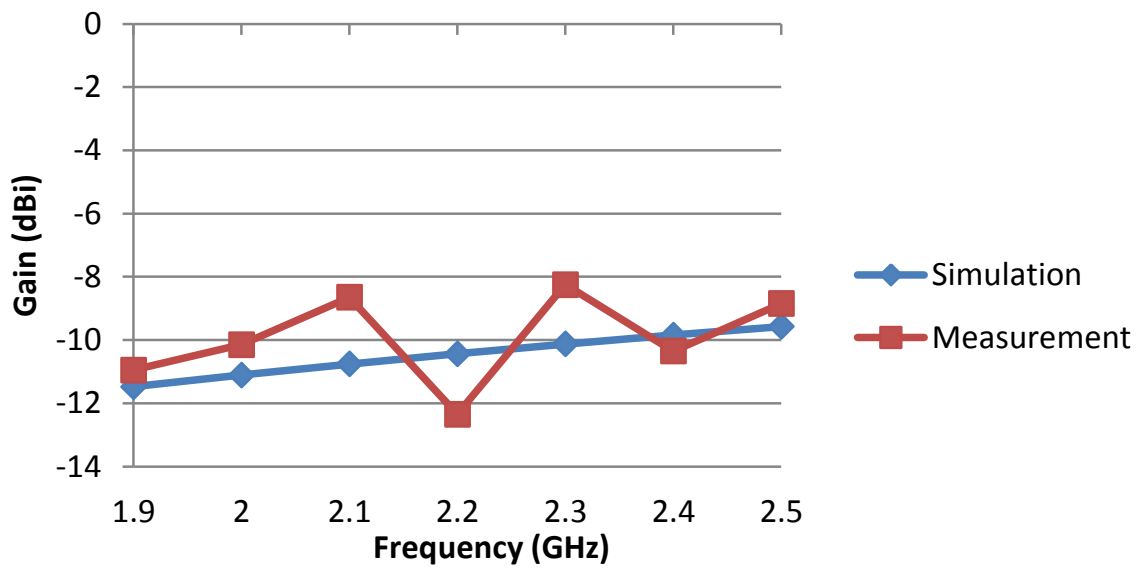

Fig 4 Measurement and Simulation result of the antenna gain

Figure 4 shows that the antenna had low gain on each frequency. That means the AUT has low efficiency on power transmission after compared with the dipole gain. The low gain was necessary because it was natural behavior of the microstrip antenna as its disadvantage. The difference result between simulation and measurement result was occurred because in the simulation, the result was estimated in an ideal condition and was obtained from absolute gain of the antenna, meanwhile in the measurement, the measured gain obtained from the relative gain comparing the AUT with the reference antenna. The reference antenna was used dipole antenna with an isotropic condition. To obtain the result that shown in the measurement devices was not easy since the value in the measurement device was oscillated. Another thing was the punctuation of the antennas, during measurement the AUT was positioned not in one line with the reference antenna.

\subsection{Directivity Measurement Result}

To measure directivity, the received power of the antenna must be measured by rotating the AUT stand holder from $\varphi=0$ until $\varphi=360$ degrees. The measurement was taken only from the frequency of $2.4 \mathrm{GHz}$ which was used for the free license frequency and Wifi. Then, the measurement result was compared with simulation results as depicted in Figure 5

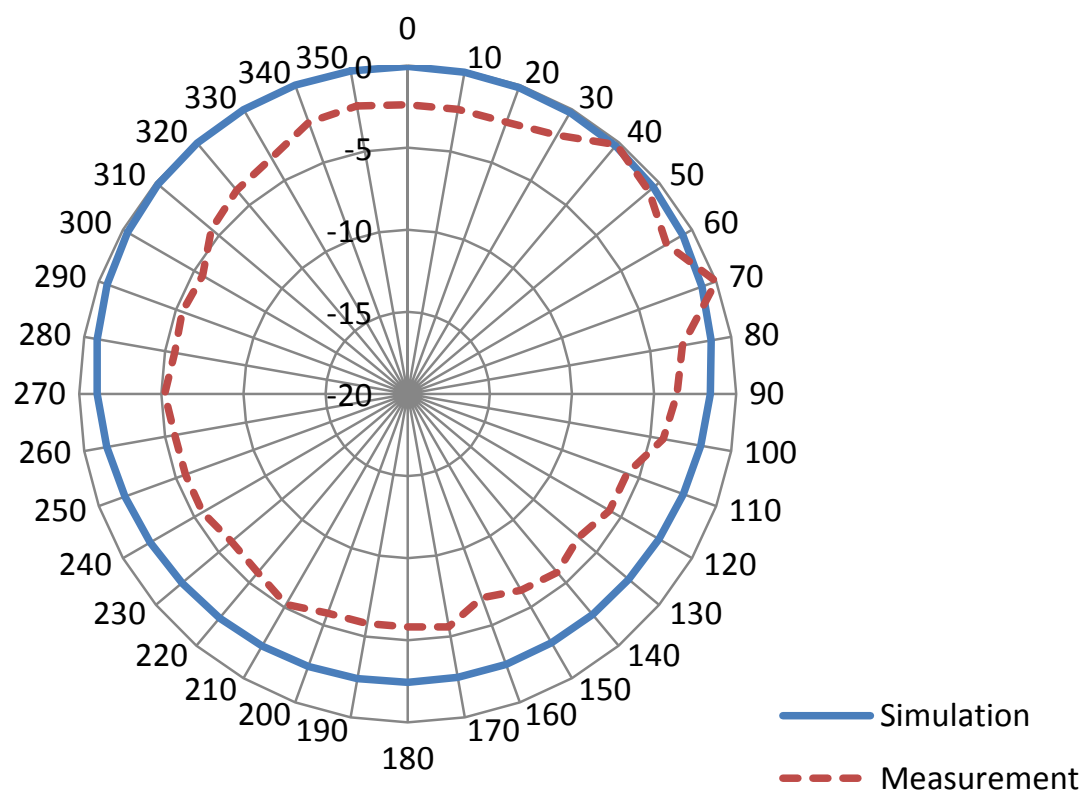

Fig 5 The antenna directivity from the measurement and simulation results

Figure 5 showed that the antenna was omnidirectional, hence the antenna transmitted power to all directions. Comparing with the simulation result, the antenna performance got the same result which was omnidirectional pattern, although the level of the accepted power between simulation and measurement was different. The differences in accepted power in the simulation and measurement were caused by the condition in 
the simulation and measurement. In the simulation, the condition around the test antenna was in ideal condition, hence there was no reflected power that was caused by the object around the antenna. The result of the simulation had level minimum accepted at the angle of 180 degrees and maximum from 340 degrees to 40 degrees that was because the accepted power followed the reference antenna.

\subsection{Axial Ratio Measurement Result}

The graphic of the axial ratio is shown in Figure 6

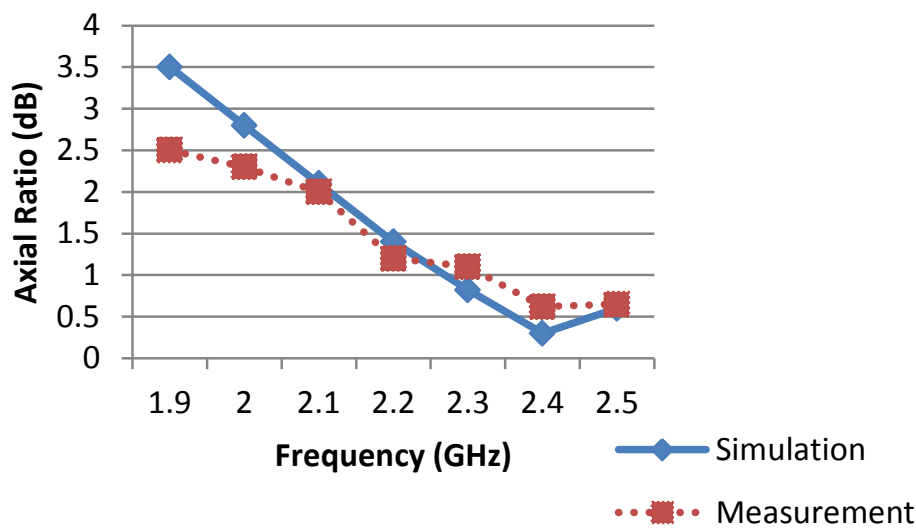

Fig 6 Axial ratio from simulation and measurement result

Figure 6 showed that the antenna was circularly polarized at range of frequency of 1.9 until $2.5 \mathrm{GHz}$. It was obtained from the axial ratios that the axial ratio value less that $3 \mathrm{~dB}$ at frequency of $1.9 \mathrm{up}$ to $2.3 \mathrm{GHz}$. The level of $3 \mathrm{~dB}$ was the tolerance of the antenna axial ratio whose circularly polarized [1].

\section{Conclusion}

Based from measurement result can be concluded that the antenna can be realized with the dimension of $1.5 \mathrm{~mm}$ in width of the microstrip line feed on the FR-4 substrate which has dimension of $0.29 \lambda_{0}$ or $40 \mathrm{~mm} \mathrm{x}$ $40 \mathrm{~mm}$ and $0.8 \mathrm{~mm}$ in thickness of the substrate. The etching process was used for antenna fabrication, which printed the copper area as proposed model on the substrate.

To reduce the misalignment between transmitting and receiving power because of the mismatched polarization was solved with the circularly polarized model fed which had two orthogonal feeding models [5][2]. The antenna had the circular polarization based on the measurement result that obtained the axial ratio level less than $3 \mathrm{~dB}$ in frequency of $1.9 \mathrm{GHz}$ up to $2.5 \mathrm{GHz}$.

The fabricated antenna worked on the frequency of 1.9 until $2.5 \mathrm{GHz}$ omnidirectionally with the low gain (less than $0 \mathrm{dBi}$ ) and has circular polarization on these frequencies. In order to avoid the deviation between simulation and measurement results, the measurement setup should be held in the anechoic chamber, not use many adapters, the punctuation of the antenna should be in line, also the measurement result get the line of sight condition which no other object between the test antenna and reference antenna.

\section{References}

[1] Balanis, C.A, 2005, Antenna theory, analysis and Design, Wiley, USA

[2] Kumar, Girish, Ray,KP, 2008, Broadband Microstrip Antennas, Artech House, UK

[3] Joseph,R ,Fukusako, T, Circularly Polarized Broadband Antenna with Circular Slot on Circular Ground-Plane, 2012, Progress in Electromagnetics Research

[4] Kim, Cheolbok, Ultra-Wideband Antenna, 2010, Intech open, p. 19

[5] Mohamed, Eman A.F, Mohamed, Darwish A.E, Design of a circularly Polarized tuning Fork Type UWB patch Antenna, 2008, Arab Academy For Science and Tecnology and Maritime Transport,

[6] Chen, Q, Zheng, H.L, Quan, T, Li, X, Broadband CPW-fed Circularly Polarized Antenna with Equiangular Tapered shaped Feedline for Ultra-Wideband Applications, 2012, Progress in Electromagnetics Research

[7] Bhartia, P, Bahl, Inder, Garg, Ramesh, Ittipiboon, 2001, Microstrip Antenna Design Handbook, Artech House, UK

[8] T. Dong and Y.P. Chen, Novel Design of Ultra-Wideband Printed Double-Sleeve Monopole Antenna, 2009, Progress in Electromagnetics Research

[9] Azim, Rezaul, Islam, Mohammad Tariqul, Misran, Norbahiah, Compact Planar Antenna for UWB Applications, 2010,World Academy of Science, Engineering and Technology 